\title{
PRESENCIA Y AUSENCIA DE MONTAIGNE EN LA OBRA DE FOUCAULT ${ }^{1}$
}

\author{
BELTRÁN JIMÉNEZ VILLAR \\ Universidad de Granada
}

\begin{abstract}
RESUMEN: A pesar de que en los trabajos de Foucault no encontramos una problematización de los Ensayos de Montaigne, a partir de las escasas menciones que hace a su pensamiento es posible reconstruir su lectura. Si Descartes es el autor que mediante un gesto violento expulsa la locura de lo razonable y clausura la tradición del ejercicio de sí reactivada en el Renacimiento, en Montaigne el ensayo surge como una técnica de continua transformación de sí ejercida por un sujeto que, ante la impotencia de la evidencia para acceder a la verdad, solo puede someterse a prueba. La relación que se establece entre ambos autores en el marco de la filosofía foucaultiana nos permite entrever cómo se relaciona el eje de la subjetividad con los del poder y el saber, así como subrayar la importancia de un estudio filosófico de los Ensayos para comprender la genealogía de la ontología del presente.
\end{abstract}

PALABRAS CLAVE: Foucault; Montaigne; Ensayos; Descartes; ejercicio de sí.

\section{Presence and Absence of Montaigne in Foucault's Work}

ABSTRACT: Despite the fact that there is not a deep study of Montaigne's Essays in Foucault's works, based on the few mentions that he makes to his thought it is possible to reconstruct his reading. If Descartes is the author who, through a violent gesture, expels the madness from the reasonable and closes the tradition of exercise of the self reactivated in the Renaissance, in Montaigne the essay emerges as a technology of constant self-transformation exercised by a subject who, faced with the impotence of evidence to access the truth, can only subject himself to test. The relationship established between the two authors within the framework of Foucaultian philosophy allows to glimpse how the axe of subjectivity is connected with the axes of power and knowledge, as well as to underline the importance of a philosophical study of the Essays in order to understand the genealogy of the ontology of the present.

KEY WORDS: Foucault; Montaigne; Essays; Descartes; exercise of the self.

Durante más de 30 años de trayectoria, los Ensayos de Montaigne nunca fueron para Foucault objeto de un estudio pormenorizado. No obstante, a pesar de ser un autor que no ocupa un lugar central en las grandes problematizaciones foucaultianas, sí es posible encontrar menciones a su pensamiento en textos de distintas épocas. Así, los objetivos de este trabajo son 1) exponer y analizar las alusiones al autor bordelés en la obra de Foucault, 2) reconstruir la posible interpretación de Montaigne que de ellas es posible extraer, y 3) mostrar la importancia del estudio filosófico de Montaigne para la genealogía de la ontología crítica de nosotros mismos.

1 Este trabajo ha sido posible gracias a la concesión de una ayuda para la formación de profesorado universitario financiada por el Ministerio de Educación, Cultura y Deporte del Gobierno de España. 


\section{Una lectura de Montaigne}

La primera aparición de Montaigne en el corpus foucaultiano se encuentra en Histoire de la folie à l'âge classique ${ }^{2}$. En esta obra el autor afirma que en la historia de Occidente se pueden detectar tres experiencias distintas de la locura: experiencia trágica, experiencia clásica y experiencia moderna. En la primera la locura se encontraba asociada a las potencias ocultas del mundo que anunciaban su consumación final. Era objeto de fascinación porque en ella residía un importante saber: el descubrimiento de un furor amenazante que revelaba la ambigua relación entre la luz y las tinieblas que dominaba el $\operatorname{cosmos}^{3}$. Este valor veritativo que la locura ostentó desde el comienzo del Renacimiento en la experiencia trágica se vio clausurado por la experiencia clásica, que comienza en el siglo XVII. En este momento la locura se transforma en lo contrario de la razón, en sinrazón, y es reducida al silencio por un «coup de force». La famosa figura de este desplazamiento es Descartes en cuyas Meditaciones Foucault reconoce el destierro de la locura del ámbito de lo razonable, lo que abre la puerta al gran encierro de los insensatos. En el camino de la duda Descartes se plantea la posibilidad de que lo engañen los sentidos o de estar dormido, sin embargo no hace lo propio con la locura ya que el mismo hecho de estar pensando excluye por principio dicha condición ${ }^{4}$. Esta nueva experiencia lleva aparejada el encierro de los locos, su internamiento no con el objetivo médico propio de la experiencia moderna (s. XIX), sino con una intención puramente «policial»: sanear el paisaje social para establecer un «orden» en la ciudad que permita el desarrollo del trabajo ${ }^{5}$.

En la lenta y progresiva transformación que llevó de la experiencia trágica a la clásica es donde la figura de Montaigne adquiere importancia. Foucault encuentra en los Ensayos un cambio fundamental con respecto a la modulación cósmica común a los grandes humanistas del Renacimiento: la locura ya no está referida a las potencias del mundo, a su verdad oculta, sino a la razón humana. Antes de que la experiencia clásica decretara el carácter inverso de la locura con respecto a la razón, la locura hubo de ser transferida del cosmos al intelecto. De esta forma, entre razón y locura se estableció una relación reversible: toda locura tiene su razón y toda razón tiene su locura. Sin embargo, en esta nueva relación, cree Foucault, ya se anuncia la victoria de la razón en la medida en que no hay mayor locura que no reconocer la propia locura. La locura se convierte así en una de las formas de la propia razón ${ }^{6}$. Y para ilustrar

2 El título de la obra en su primera edición de 1961 fue Folie et déraison à l'âge classique. A partir de la edición de 1972, pasa a denominarse Histoire de la folie à l'âge classique. Dado que el texto no sufre variaciones relevantes para el presente estudio, se empleará esta última versión: Foucault, M., Histoire de la folie à l'âge classique, Gallimard, París 1972.

3 Ibid., pp. 32-33.

4 Ibid., pp. 56-58.

5 Ibid., p. 74.

6 Ibid., pp. 41-44. 
ambas ideas Foucault se sirve de los Ensayos. En primer lugar recoge la escena en la que Montaigne visita al poeta Tasso, delirante a pesar de su excelsa capacidad, lo que le lleva a entrever una estrecha «vecindad» entre razón y locura, y a preguntarse si no ha sido precisamente cegado por una «claridad»:

Visitant le Tasse en son délire, Montaigne éprouve dépit plus encore que pitié; mais admiration, au fond, plus encore que tout. Dépit, sans doute, de voir que la raison, là même où elle peut atteindre ses sommets, est infiniment proche de la plus profonde folie : "Qui ne sait combien est imperceptible le voisinage d'entre la folie avec les gaillardes élévations d'un esprit libre, et les effets d'une vertu suprême et extraordinaire?». Mais il y a là sujet à paradoxale admiration. Car n'est-ce pas le signe que de cette même folie, la raison tirait les ressources les plus étranges. Si le Tasse, «l'un des plus judicieux, ingénieux et plus formé à l'air de cette antique et pure poésie qu'autre poète italien ait jamais été», se trouve maintenant «en si piteux état, survivant à soi-même», ne le doit-il pas à "cette sienne vivacité meurtrière? à cette clarté qui l'a aveuglé?» [...] Si la folie vient sanctionner l'effort de la raison, c'est que déjà elle faisait partie de cet effort [...] Il n'y a pas de forte raison qui ne doive se risquer dans la folie pour parvenir au terme de son œuvre, «point de grand esprit, sans mélange de folie... C'est en ce sens que les sages et les plus braves poètes ont approuvé de folier et sortir des gonds quelquefois». La folie est un dur moment, mais essentiel, dans le labeur de la raison; à travers elle, et même dans ses apparentes victoires, la raison se manifeste et triomphe. La folie n'était, pour elle, que sa force vive et secrète ${ }^{7}$.

Y como consecuencia de lo anterior, no hay mayor sinrazón que no reconocer la propia locura, la debilidad constitutiva de la razón. Cita Foucault a Montaigne:

«La présomption est notre maladie naturelle et originelle. La plus calamiteuse et fragile de toutes les créatures, c'est l'homme, et quant la plus orgueilleuse. Elle se sent et se voit logée ici par la bourbe et la fiente du monde, attachée et clouée à la pire, plus morte et croupie partie de l'univers, au dernier étage du logis et le plus éloigné de la voûte céleste, avec les animaux de la pire condition des trois, et va se plantant par imagination au-dessus du cercle de la lune et ramenant le ciel sous ses pieds. C'est par la vanité de cette même imagination qu'il égale à Dieu». Telle est la pire folie de l'homme; ne pas reconnaître la misère où il est enfermé, la faiblesse qui l'empêche d'accéder au vrai et au bien; ne pas savoir quelle part de folie est la sienne. Refuser cette déraison qui est le signe même de sa condition, c'est se priver d'user jamais raisonnablement de sa raison ${ }^{8}$.

Ahora bien, si Montaigne se encuentra más allá de la experiencia trágica, también se encuentra más acá de la experiencia clásica. A pesar de que en los Ensayos la locura haya pasado a entablar una relación reversible con la razón, e incluso a ser una de sus formas, «[q]uand Montaigne rencontrait le Tasse, rien

7 Ibid., pp. 45-56.

$8 \quad$ Ibíd., p. 44. 
ne l'assurait que toute pensée n'était pas hantée de déraison»`. Es precisamente el «coup de force» cartesiano que abre la experiencia clásica al instaurar una relación mutuamente excluyente entre locura y pensamiento el que no se encuentra en Montaigne. La experiencia de una razón irrazonable o una sinrazón razonable, propia del Renacimiento, es clausurada en el siglo XVII:

La Non-Raison du XVIe siècle formait une sorte de péril ouvert dont les menaces pouvaient toujours, en droit au moins, compromettre les rapports de la subjectivité et de la vérité. Le cheminement du doute cartésien semble témoigner qu'au XVIIe siècle le danger se trouve conjuré et que la folie est placée hors du domaine d'appartenance où le sujet détient ses droits à la vérité. [...] Entre Montaigne et Descartes un événement s'est passé : quelque chose qui concerne l'avènement d'une ratio ${ }^{10}$.

El autor de los Ensayos aparece como una figura de transición en la que despunta una "conciencia crítica» propia del Renacimiento que va a facilitar el desplazamiento desde la experiencia trágica de la locura a la experiencia clásica $^{11}$. Desplaza la locura de la verdad del mundo al discurso de los seres humanos y la sitúa en una relación siempre abierta con la razón. No obstante, la tragedia deja paso a la dialéctica ${ }^{12}$ en tanto que el sabio tiene la última palabra para describir la razón irrazonable o denunciar la razón presuntuosa ${ }^{13}$.

En un texto del mismo año dedicado a Révolution astronomique de Alexandre Koyré, Foucault vuelve a mencionar a Montaigne. Alaba esta obra por mostrar los tortuosos caminos que dieron lugar a los hitos de las revoluciones astronómicas, donde se dieron fuertes intercambios e influencias recíprocas entre lo verdadero y lo falso. Apoya la lectura que el autor hace de Kepler, para quien no era posible la enunciación de una verdad sin el relato del proceso teñido de errores y vestigios del pasado que había llevado hasta ella, e indica que esto lo convierte en una figura intermedia entre Montaigne y Descartes. El primero fue un escéptico que levanta acta del error, y el segundo encuentra un método infalible para un nuevo comienzo absoluto. A este respecto son especialmente interesantes las palabras de Foucault: «Montaigne perdait les pistes et savait qu'il les perdait. Descartes, d'un geste, regroupe toutes les erreurs possibles,

$9 \quad$ Ibid., p. 57.

$10 \quad$ Ibid., p. 58.

11 La conciencia crítica de la locura nace en el Renacimiento y se convierte en una de las cuatro formas de conciencia que, en sus distintas relaciones y modulaciones, constituyen las experiencias de la locura clásica y moderna. Véase Ibíd., pp. 182-186. Guerrier ha sostenido que la razón del desinterés de Foucault por la obra de Montaigne se debe a su localización del lado de la experiencia crítica, una de las primeras afrentas que sufrió la locura, $c f r$. GueRrier, O., «Michel Foucault et Montaigne: affinités et évitement», en: Panichi, N., RagGhianti, R., y SAvorelli, A., (eds.), Montaigne contemporaneo, Edizioni Della Normale, Pisa 2011, pp. 259272. Por otro lado, investigaciones recientes han puesto en duda la lectura foucaultiana de la locura en Montaigne, $c f r$. Morice, J., «Montaigne et la folie» en: Montaigne Studies, 26, 2014, pp. 177-193.

12 Foucault, M., Histoire de la folie à l'âge classique, p. 187.

13 Ibid., p. 39. 
en fait une grosse liasse essentielle, la traite impatiemment comme le fonds diabolique de tous les dangers éventuels: puis se considère quitte» ${ }^{14}$. En estas palabras es evidente el eco de Histoire de la folie. Descartes se siente a salvo al formular el cogito, una primera verdad de la que es imposible no estar cierto, pero Foucault denuncia que aquí se esconde un gesto violento por el que la locura queda excluida por principio del procedimiento de la duda metódica. Mientras que los errores y las ilusiones son superadas por la estructura de la verdad que se impone, es el sujeto que piensa el que excluye la locura ${ }^{15}$. Sin embargo, Montaigne es consciente de no poder resistirse a su propia desorientación, la ambigüedad constitutiva de la razón lo condena a la intemperie.

Con posterioridad a la lectura de Montaigne asociada al problema de la locura, en otros tres textos de esta década volvemos a ver aparecer a Montaigne pero con una importancia menor. En Les mots qui saignent es nombrado como una de las principales figuras de la lengua francesa ${ }^{16}$, y en Les mots et les choses se menciona su concepción del lenguaje como representativa de la episteme renacentista ${ }^{17}$. En el mismo año, en el marco de una entrevista centrada en la crítica al humanismo que acaba de emprender, Foucault afirma que la idea extendida de que este comienza con Montaigne es una ilusión retrospectiva. No es hasta el final del siglo XVIII cuando el hombre se convierte en objeto de conocimiento $^{18}$.

Después del primer acercamiento a Montaigne, centrado en su relación con Descartes a colación del problema de las relaciones entre razón y locura, habrá que esperar casi dos décadas para que el autor de los Ensayos vuelva a ser objeto de la reflexión de Foucault. Es el desplazamiento que emprende en su obra a partir del año 1980 el que va a permitir una nueva exploración. Tras el estudio de las formas en las que el sujeto es objeto de saber y de dominación, Foucault arriba a la noción de gobierno en la que descubre una cohabitación entre procesos de coerción y técnicas mediante las que el individuo se constituye o se modifica a sí mismo. Haciendo la genealogía de las exhortaciones a decir la verdad sobre uno mismo que forman parte de las técnicas de gobierno de los otros, Foucault toma consciencia de que estas no siempre han estado enmarcadas bajo una obligación general de obediencia, sino que en el mundo griego y romano han permitido el establecimiento de una relación del sujeto consigo mismo en la que este se constituía como tal. Esto lleva a Foucault a afirmar que el sujeto no solo es constituido a través de las prácticas de dominación, sino

14 Foucault, M., «Alexandre Koyré: La Révolution astronomique, Copernic, Kepler, Borelli», en: Dits et écrits (vol. I), Gallimard, París 1994, p. 171.

15 Foucault, M., Histoire de la folie à l'âge classique, p. 57.

16 Foucault, M., «Les mots qui saignent», en: Dits et écrits (vol. I), pp. 424-426.

17 Foucault, M., Les mots et les choses, Gallimard, París 1966, p. 55.

18 Foucault, M., «L'homme est-il mort?», en: Dits et écrits (vol. I), p. 540. 
que también se constituye a sí mismo a través de ciertos métodos ${ }^{19}$. Será la investigación de estas técnicas de sí la que permita la aparición de Montaigne.

En este contexto, el curso del Collège de France L'herméneutique du soi marca nuestra siguiente parada. Aquí Foucault tiene como objetivo demostrar que, a diferencia de lo que la tradición ha hecho creer, en la cultura griega el precepto délfico ghtohi seauton (conócete a ti mismo) estaba subordinado a la noción más general de epimeleia heautou (inquietud de sí). La necesidad del autoconocimiento aparece con el personaje de Sócrates siempre asociada con el principio más general de ocuparse de uno mismo, como una de sus formas $^{20}$. Es decir, el sujeto solo podía tener acceso a la verdad bajo la condición de realizar previamente sobre sí mismo en tanto que sujeto una serie de transformaciones ${ }^{21}$. Dados estos presupuestos Foucault emprende un recorrido por las distintas variaciones de esta noción en el que distingue tres momentos: el socrático-platónico, la edad de oro del epimeleia heautou en el periodo helenístico y romano, y la ascética del cristianismo primitivo. Ahora bien, si esta noción es tan extraña para nosotros es debido, afirma el autor, a los fuertes golpes que la han condenado al olvido. El primero de ellos fue precisamente el ascetismo cristiano que transformó el objetivo de la constitución de sí griega y romana en la necesidad de renunciar a sí mismo para alcanzar la santidad. En consecuencia, el horizonte general del sí mismo comenzó a desdibujarse. Pero para Foucault el punto determinante de esta decadencia es el «momento cartesiano" que revalorizó el autoconocimiento y devaluó el cuidado de sí. Paradójicamente Descartes utiliza una técnica de sí como la meditación, originalmente empleada para obrar una transformación del sí mismo que permitiera el acceso a la verdad, con el fin de establecer como primera verdad la evidencia de la existencia del sujeto. En otras palabras, el individuo no necesita poner en marcha ninguna técnica de sí que transforme su ser en tanto que sujeto para acceder a la verdad, le basta con el conocimiento ${ }^{22}$. Y aunque se encuentran algunas resonancias de la tradición de los ejercicios de sí en autores posteriores como Leibniz o Spinoza y a pesar de que esta cuestión se desplazó al interior de ciertas formas sociales - como la necesidad de pertenecer a una clase-, la importancia del momento cartesiano reside en que fue el primer paso del proceso que condenó al olvido a las relaciones entre el sujeto y la verdad en la cultura occidental ${ }^{23}$.

Teniendo en cuenta la torsión del cristianismo primitivo y la posterior exclusión cartesiana, Foucault afirma:

19 Foucault, M., L'origine de l'herméneutique de soi. Conférences prononcées à Dartmouth College, 1980, Vrin, París 2013, pp. 37-40.

${ }_{20}$ Foucault, M., L'herméneutique du sujet. Cours au Collège de France (1981-1982), Gallimard, París 2001, pp. 5-7

21 Ibid., p. 17.

22 Ibid., pp. 16-17.

23 Ibid., pp. 29-31. 
[Je] crois qu'il faut aussi remarquer que le thème du retour à soi a sans doute été, à partir du XVIe siècle, un thème récurrent dans la culture «moderne». Mais je crois qu'on ne peut pas ne pas être frappé, aussi, du fait que ce thème du retour à soi a été au fond reconstitué - mais par fragments, par bribes - dans une série d'essais successifs qui ne se sont jamais organisés sur un mode aussi global et continu que dans l'Antiquité hellénistique et romaine. [...] Bien sûr, vous trouvez au XVIe siècle toute une éthique de soi, toute une esthétique aussi de soi, qui est d'ailleurs très explicitement référée à celle qu'on trouvait chez les auteurs grecs et latins dont je vous parle. Je pense qu'il faudrait relire Montaigne dans cette perspectivelà, comme une tentative de reconstituer une esthétique et une éthique du soi $^{24}$.

La necesidad de releer los Ensayos de Montaigne desde la perspectiva de un ejercicio de sí, es decir, no bajo el paradigma del autoconocimiento, sino como el de una práctica de transformación de sí en tanto que sujeto, es reiterada por Foucault en el debate que mantuvo en Berkeley en 1983 en el Departamento de Francés. A la pregunta de cuál es el lugar de las técnicas de sí en la reactivación de la cultura antigua en el siglo XVI, el autor responde que, en el marco de la gran crisis religiosa que se desata a finales del siglo XV se desarrollaron nuevas formas de relación consigo mismo en oposición a las prácticas de la pastoral cristiana como la confesión. Y en dicha búsqueda de nuevas relaciones:

[I]l me semble même que la notion d'essai chez Montaigne, d'essai à prendre au sens strict, c'est-à-dire d'épreuve —on se mesure à ce qu'il faut, on voit si, on voit jusqu'où on peut, etc.-, cette notion en quelque sorte d'épreuve de soi-même me paraît assez proche de cette thématique que l'on trouve évidemment chez les stoïciens, et où l'examen de soi n'est pas tellement fait pour découvrir une forme cachée au fond de soi-même, une vérité cachée au fond de soi-même, mais [est] une tentative pour savoir, [pour] faire l'épreuve de ce qu'on sait et de ce qu'on ne sait pas, de ce qu'on peut et de ce qu'on ne peut pas, de la liberté dont on dispose et de la dépendance, des dépendances par lesquelles on est encore attaché. L'épreuve de soi plus le déchiffrement de soi m'apparaissent assez évidents chez Montaigne, mais je me trompe peut-être ${ }^{25}$.

En otras palabras, las famosas afirmaciones de los Ensayos «el yo es el tema de mi libro», "yo soy mi física, mi metafísica», "un libro consustancial a su autor», no han de ser leídas como descripciones de una investigación autocognoscitiva dirigida al desciframiento interior del sujeto. Antes bien, mediante la escritura de sí que pone en obra, Montaigne se somete a prueba en tanto que sujeto de acciones posibles con el objetivo de transformarse. Por esta razón, tanto las estructuras retóricas como el conocimiento de sí que pueda encontrarse en los Ensayos han de ser subordinados a la empresa general de transformar el saber en ethos ${ }^{26}$.

24 Ibid., p. 240. 168-169.

25 Foucault, M., Qu'est-ce que la critique? Suivi de La culture de soi, Vrin, París 2015, pp.

${ }_{26}$ Ibíd., p. 163. Para una profundización en la transformación del saber en ethos véase Foucault, M., L'herméneutique du sujet. Cours au Collège de France (1981-1982), pp. 228-229. 
Además la reactivación de la cultura de sí que tiene lugar en el Renacimiento y de la que Montaigne es un claro ejemplo está relacionada con el momento cartesiano ya apuntado. Foucault afirma que la relación entre Montaigne, que reactiva la práctica de sí, Pascal y Descartes ha de ser estudiada desde esta nueva perspectiva. Así, en primer lugar, Pascal no solo es un crítico de Montaigne - a nivel teórico-, sino que se sitúa en una tradición distinta: en el rechazo de la pertinencia de la transformación de sí $^{27}$. Y en segundo lugar, Descartes llevará a cabo la torsión mencionada por la que, gracias a una meditación, sustituye un sujeto constituido a través de unas prácticas de sí por un sujeto fundador de una práctica de conocimiento ${ }^{28}$. Es decir, se sirve de un ejercicio de sí para establecer las condiciones que hacen posible el conocimiento y la irrelevancia de todo ejercicio de sí posterior. De esta forma, en el umbral de la Modernidad se halla una ruptura que se explicita en la diferencia entre Montaigne y Descartes. Mientras que en el primero el sujeto no es capaz de acceder a la verdad si no opera sobre sí previamente un cierto trabajo que lo hacía susceptible de ello - y que se pone en acto en los Ensayos-, en el segundo la evidencia ha sustituido a la práctica de sí como punto de unión de la relación entre sujeto y la verdad: es suficiente la verdad evidente de lo que veo para que acceda a ella definitivamente. Y como consecuencia de la aparición del sujeto de conocimiento no ascético, la ciencia podrá institucionalizarse ${ }^{29}$.

Aquí termina la exigua presencia de Montaigne en la obra de Foucault. Pero a pesar de ser escasa, está en relación con dos importantes núcleos temáticos de su pensamiento, como son la locura y el cuidado de sí, y con dos momentos de ruptura que en ambos casos representa la figura de Descartes. Cabe preguntarse ahora si existe alguna relación entre ambas transformaciones, si puede establecerse un nexo entre los papeles que Montaigne representa en ellas y qué relevancia puede tener esta reflexión para una comprensión del proyecto foucaltiano.

\section{Montaigne y las transformaciones de Los COUPS CartéSIENS}

Como ya se ha indicado, en Histoire de la folie Foucault describe el efecto de la filosofía cartesiana en la comprensión de la locura como un «coup de force» ${ }^{30}$, un gesto violento por el que el pensamiento y la locura se convierten en elementos inmiscibles. Por otra parte, en el curso de 1982 el significado de la torsión cartesiana por la que el sujeto deja de ser constituido para ser constituyente de una práctica de conocimiento es denominada «moment cartésien» ${ }^{31}$. No obstante, la expresión «coup cartésien» es recuperada en el debate de Berkeley en

27 Foucault, M., Qu'est-ce que la critique? Suivi de La culture de soi, p. 175.

28 Ibíd.

29 Ibíd., p.176.

30 Foucault, M., Histoire de la folie à l'âge classique, p. 56.

31 Foucault, M., L'herméneutique du sujet. Cours au Collège de France (1981-1982), pp. 15-16, 19, 29. 
la respuesta sobre las relaciones entre Montaigne y Descartes en el marco de la epimeleia heautou ${ }^{32}$. Foucault da algunas pistas para entender cómo pueden estar relacionados estos dos momentos de ruptura. En L'herméneutique du sujet el autor analiza el cisma con respecto a las técnicas espirituales que representa Descartes y subraya que, a partir de entonces, las condiciones de acceso de la verdad no atañen a transformaciones que haya que realizar sobre el sujeto. En su lugar se encuentran condiciones internas al acto de conocimiento - formales, objetivas, metodológicas- y otras extrínsecas que solo conciernen al sujeto en su existencia concreta pero no en cuanto a la estructura de su subjetividad, como son las condiciones culturales o morales - recibir formación o no tener intereses espurios-. Y entre estas últimas hay una especialmente interesante para nuestro propósito: «conditions comme: "Il ne faut pas être fou pour connaitre la vérité" (importance de ce moment chez Descartes)» ${ }^{33}$. Pero, si de acuerdo con Histoire de la folie Descartes había exiliado a la locura al plantear que su posibilidad era ajena al pensamiento, ¿qué amenaza debe conjurar el sujeto concreto para conocer la verdad? La locura en la experiencia clásica puede afectar al individuo pero no al pensamiento, es decir a la razón $^{34}$. En este sentido, nos es muy valiosa la diferencia que señala Fréderic Gros entre locura y sinrazón, a pesar de las ambigüedades que encontramos en el texto foucaultiano. La primera refiere a la «división originaria» de la que Foucault busca realizar una «historia trágica» de la cultura, en la que observar cómo esta se construye por el rechazo de lo que no es. De esta forma la locura significa el límite de toda experiencia posible. Después, en un nivel superior, se construyen las experiencias que de esta división, de este límite que representa la locura, tienen lugar en cada época. Y precisamente es la «sinrazón» la que define la experiencia clásica, aunque Foucault no sea exhaustivo en su uso ${ }^{35}$. A este respecto es particularmente relevante la precisión que ofrece el autor en su respuesta a la crítica formulada por Derrida ${ }^{36}$ : Descartes se niega a someterse

32 Foucault, M., Qu'est-ce que la critique? Suivi de La culture de soi, pp. 175-176.

33 Foucault, M., L'herméneutique du sujet. Cours au Collège de France (1981-1982), p. 19. El autor matiza su posición sobre Descartes en una entrevista posterior. No se trata de que a partir de las Meditaciones ya no sea necesaria ninguna modificación del ser del sujeto para acceder a la verdad, sino que dicha transformación está enteramente determinada por el conocimiento. El sujeto capaz de verdad es el sujeto capaz de conocer y de esta forma la inquietud de sí se subordina al ideal de funcionamiento de la ciencia. Cfr. Foucault, M., "L'éthique du souci de soi comme pratique de la liberté», en: Dits et écrits (vol. IV), Gallimard, París 1994, p. 723.

34 Foucault., M., Histoire de la folie à l'âge classique, p. 58.

35 Gros, F., Foucault y la locura (H. Pons, trad.), Nueva Visión, Buenos Aires 2000, pp. 31-33.

36 El famoso debate entre Foucault y Derrida ha ocupado a numerosos especialistas hasta el momento. A modo de ejemplo de las últimas derivas que esta discusión ha suscitado $c f r$. Barroso Fernández, Ó., "Ni trazas textuales, ni prácticas discursivas. Suárez y el barroco como claves de comprensión de la duda cartesiana» en: Ingenium. Revista Electrónica de Pensamiento Moderno y Metodología en Historia de las Ideas, 11, 2017, pp. 29-46; Revel, J., Un malentendu philosophique. Foucault, Derrida et l'affaire Descartes, Bayard Culture, París 2015. 
a la prueba de la locura y para ello define a los locos como insani, amens y demens. El primer término es una expresión médica que describe las imaginaciones de las que son víctimas los insensatos y que les llevan a tomarse por lo que no son. Los otros dos conceptos pertenecen al campo jurídico, designan la incapacidad de estos individuos para ejercer ciertos derechos y Descartes los utiliza para justificar la imposibilidad de seguir su ejemplo ${ }^{37}$. La prueba de la locura no puede ser efectuada porque en ese instante la meditación quedaría suspendida por la invalidez del sujeto que se propone realizarla. En cambio, el sueño sí le permite dudar de su actualidad — del lugar en el que está o del calor que la estufa le hace sentir- sin que por ello deje de ser un sujeto válido, ya que forma parte de los recuerdos y virtualidades más frecuentes ${ }^{38}$. Finalmente, la hipótesis genio maligno, a pesar de que extiende la sospecha mucho más allá de los errores sensibles de los que son objeto los locos, quien la formula no es por ello preso de su efecto: la locura se impone, el gran engañador me permite no creer que esta actualidad es la mía. Foucault afirma que es la constitución previa del sujeto razonable mediante la expulsión de la locura la que permite que de hecho no se le preste ninguna credulidad al genio maligno ${ }^{39}$. Es decir, a juicio de Foucault, la descalificación de la locura como posibilidad para un sujeto razonable es previa a la meditación. De esta forma, el ejercicio de Descartes ya ha sellado la oquedad de la locura cuando se plantea dudar de todo lo que alguna vez ha sido tomado por verdadero y de esta forma también ha definido con anterioridad lo que significa pensar.

Por tanto, los dos coups cartésiens que Foucault denuncia en la exclusión de la locura y en el olvido de la tradición de la epimeleia heautou están estrechamente relacionados. Descartes utiliza la meditación, un ejercicio del pensamiento propio de la tradición de la inquietud de sí en el que el sujeto se modifica a sí mismo mediante el discurso que va pronunciando, con el pretexto de someter a duda todo lo que ha sido tomado alguna vez por verdadero y fundamentar así el conocimiento científico, pero sin confesar que su gran victoria ha sido previa, ya que la razón entra en el proceso purificada del peligro que quería afrontar. Al rechazar la legitimidad de la prueba de la locura, que ponía en duda lo más evidente, establece implícitamente el límite de la verdad y de lo razonable: lo evidente es verdadero ${ }^{40}$. Como consecuencia de ello, este ejercicio de sí acotado y controlado llega a la conclusión de que el sujeto puede alcanzar la verdad mediante la correcta aplicación de los criterios del recto conocimiento. Las técnicas utilizadas durante siglos para transformar el ser del sujeto se convierten en métodos para la fundamentación del conocimiento

37 Foucault, M., «Mon corps, ce papier, ce feu», en: Dits et écrits (vol. II), Gallimard, París 1994, pp. 253-254.

38 Ibíd., p. 261.

39 Ibíd., p. 266.

40 Foucault, M., Du gouvernement des vivants. Cours au Collège de France (1979-1980), Gallimard, París 2012, p. 96. 
científico ${ }^{41}$. El gesto violento que expulsa a la locura permite que la meditación termine consagrando al sujeto cognoscente como sujeto verdadero y someta la tradición del epimeleia heautou al conocimiento.

Llegado este punto, para comprender el papel de Montaigne con respecto a estos momentos cartesianos es necesario acudir al contexto en el que Foucault emprende el estudio de la hermenéutica de sí. En un texto adjuntado a la conferencia Tecnología de sí de 1982, el autor indica que ha sido la investigación sobre la "gubernamentalidad» la que le ha llevado a esta nueva problematización. Su objetivo, afirma, ha sido el estudio de una historia de los diferentes modos que han permitido a los seres humanos elaborar un conocimiento sobre sí mismos, como son la psiquiatría, la sexualidad o las instituciones penales. Ahora bien, su análisis no buscaba desvelar si se trataba de discursos ideológicos o de si tenían una importancia económica, sino analizarlos como juegos de verdad ligados a técnicas particulares que los seres humanos emplean consigo mismos. Y estas técnicas son de cuatro tipos: tecnologías de producción, tecnologías de significación, tecnologías de dominación, y técnicas de sí. Estas cuatro tecnologías no funcionan casi nunca de forma separada y Foucault confiesa que su estudio se ha centrado en las dos últimas: las de dominación y las de sí. Por esta razón ha trabajado en la producción de una historia del saber y de las organizaciones del saber relacionadas con los procesos de dominación y las técnicas de sí. En este sentido y con respecto a la locura, su intención ha sido mostrar cómo el tipo de dirección de los individuos en el interior y en el exterior del asilo ha permitido la construcción del discurso psiquiátrico. Sin embargo, después de haber prestado gran atención a las técnicas de dominación, cada vez se ha interesado más en los puntos de interacción entre ambas, donde la forma en la que los individuos son dirigidos se articula sobre la forma en la que ellos se conducen a sí mismo, lo que ha denominado "gubernamentalidad» ${ }^{42}$. Pero, ¿qué tipo de articulación es esta? ¿Qué tipo relación entablan estos dos ámbitos? Foucault afirma que en estos puntos de contacto, las técnicas de dominación «han recurrido» a los procesos que los individuos utilizan para transformarse a sí mismos ${ }^{43}$. ¿Qué implica este recurso?

En la investigación de las técnicas de sí Foucault centra su atención en dos grandes periodos: la época helenística y romana, en la que entrevé la aparición de una "cultura de sí», y el cristianismo primitivo, que siguiendo sus líneas fundamentales la transforma ${ }^{44}$. Ambas son formas de espiritualidad, es decir, en ellas el sujeto accede a la verdad como efecto de contragolpe de la conversión que obra sobre sí a través de unas prácticas ${ }^{45}$. Sin embargo, el desplazamiento

41 Foucault, M., L'origine de l'herméneutique de soi. Conférences prononcées à Dartmouth College, 1980, pp. 119-121.

42 Foucault, M., Dire vrai sur soi-même. Conférences prononcées à l'Université Victoria de Toronto, 1982, Vrin, París 2017, pp. 30-31.

$43 \quad I b i d$, p. 31. La traducción es nuestra.

44 Foucault, M., L'herméneutique du soi. Cours au Collège de France (1981-1982), p. 172.

45 Ibid., p. 17. 
que se produce entre la filosofía antigua y el primer cristianismo consiste en que el tipo de conversión que perseguían las escuelas filosóficas es transformado. En la cultura de sí, la necesidad de ocuparse de sí denota una forma de vida en la que se busca establecer una serie de relaciones consigo mismo de posesión y soberanía, gracias a la adquisición de un equipamiento que permite afrontar la vida y el mundo — de ahí su función crítica y terapéutica-. En cambio, en el cristianismo primitivo, a pesar de que los ejercicios antiguos son transferidos a las nuevas prácticas monásticas, su fin ya no es la constitución de una adecuada relación consigo mismo que posibilite la vida, sino la renuncia de sí y la separación con respecto al mundo para abismarse en Dios ${ }^{46}$. Por tanto, mientras que en la cultura de sí la autoconstitución del yo es la meta, en la espiritualidad cristiana el yo renuncia a ser sujeto de la voluntad ya que solo será capaz de verdad cuando desaparezca ${ }^{47}$. De hecho, fue el estudio de la confesión en el cristianismo primitivo, en el marco de la genealogía de la sexualidad, el que llevó a Foucault a la conclusión de que la obligación general de decirlo todo provenía del cuidado de sí antiguo ${ }^{48}$. Pero, ¿qué significado tiene este desplazamiento? El cristianismo primitivo se sirvió de una técnica destinada a la constitución de sí, a la consecución de la autonomía del sujeto, para instaurar una relación de obediencia en la que la renuncia de sí era la condición de posibilidad que permitía alcanzar la santidad.

En el primer cristianismo la penitencia es un estatus general de la existencia que evita la expulsión de la comunidad de un cristiano que ha pecado y que permite su reintegración. Entre sus obligaciones se encontraba la necesidad de manifestar la verdad a través de expresiones corporales y simbólicas, poner en práctica una revelación dramática del rechazo de sí que hiciera posible el perdón, lo que los padres de la Iglesia denominan exomologesis ${ }^{49}$. Ahora bien, con el monacato de los siglos IV y V se produce el entrecruzamiento de las técnicas de existencia filosófica y la penitencia. El autosacrificio de sí, siguiendo el modelo del martirio, se sirve del lenguaje como medio para la manifestación de la verdad. La renuncia de sí solo puede llevarse a cabo mediante un tipo de relación de obediencia con el otro que pasa por la obligación de decir veraz sobre uno mismo. La dirección de conciencia en la Antigüedad se desarrollaba bajo el modelo pedagógico: una enseñanza provisional por parte de un maestro competente que enseñaba una verdad — que a la vez era una regla de comportamiento- que nos hacía capaces de autogobernarnos. En el monacato la necesidad de la dirección es permanente y su validez no depende ni de la orden ni del estatus de quien la ordena, sino del mero hecho de obedecer. La

46 Foucault, M., Dire vrai sur soi-même. Conférences prononcées à l'Université Victoria de Toronto, 1982, pp. 93-94.

47 Foucault, M., L'origine de l'herméneutique de soi. Conférences prononcées à Dartmouth College, 1980, pp. 87-88.

48 Foucault, M., Discours et vérité. Précèdé de La parrêsia, Vrin, París 2016, p. 26.

49 Foucault, M. L'origine de l'herméneutique de soi. Conférences prononcées à Dartmouth College, 1980, pp. 74-76. 
santidad se alcanza mediante las virtudes de la humildad (estimar que pueden darnos órdenes), la paciencia (no oponer resistencia) y la sumisión. Y para que la obediencia permee toda la vida es imperativo decir todos los movimientos de nuestro pensamiento para discernir si la fuente de la que provienen es espuria $^{50}$. Posteriormente, entre los siglos VI y VII se produce una contaminación recíproca entre la revelación dramática de sí —exomologesis - y la obligación de decir la verdad sobre sí mismo - exagoreusis - que va a conducir a que la dirección de conciencia deje de ser una práctica reservada a las comunidades monásticas y que se generalice en tanto que función pastoral. Mientras que en los conventos se estableció una reglamentación rigurosa de faltas y sanciones, la comunidad de los laicos se conformó bajo una relación de dirección con el obispo. Y a partir del siglo VII, Foucault documenta una tercera mutación en este proceso que conduce a la penitencia tarifada en la que se establece una proporción calculada entre faltas y sanciones ${ }^{51}$. En conclusión, para el autor lo relevante es cómo una técnica que formaba parte indispensable de la práctica filosófica entendida como arte de existencia y que tenía como fin la consecución de la autonomía del individuo se convierte en la pieza que permite construir una forma de ejercer el poder basada en la obediencia y la renuncia de sí como el poder pastoral ${ }^{52}$.

Si para Foucault la experiencia la constituyen los ejes del saber, del poder y de la relación de sí consigo, la transformación de la obligación del decir veraz en la ascesis cristiana implica su captura por el régimen de poder pastoral. En esto consiste el mencionado «recurso» que las técnicas de dominación hacen de las técnicas de sí. Como Gilles Deleuze ha mostrado magistralmente, el poder no ha cesado en su intento de reconquistar las innovaciones que se producen en este eje de la subjetivación, ni el saber ha dejado de investir estas tentativas. Con los griegos comienza la historia de cómo el poder ha buscado apropiarse de los procesos de subjetivación que lo exceden ${ }^{53}$, y a la vez de cómo nuevas formas de subjetivación no han dejado de dibujarse como respuesta a este movimiento constante ${ }^{54}$. A este respecto es de especial relevancia la expresión «ética agonal» acuñada por Wilhelm Schmid para definir la propuesta foucaultiana. En la medida en que entre las relaciones de poder y la «intransitividad de la libertad» hay una relación agónica, el sentido de la ética entendida como arte de vivir reside en la búsqueda perenne de la apertura que impida la solidificación de las relaciones de poder y de saber ${ }^{55}$.

50 Foucault, M. Mal faire, dire vrai. Fonction de l'aveu en justice. Cours de Louvain, 1981, Presses Universitaires de Louvain, Louvain-la-Neuve 2012, pp. 127-146.

51 Ibid., pp. 175-176.

52 Foucault, M., Histoire de la sexualité IV. Les aveux de la chair, Gallimard, París 2018, pp. 389-390.

53 Deleuze, G., La subjetivación: curso sobre Foucault. Tomo III (P. Ires y S. Puente trad.), Cactus, Buenos Aires 2015, p. 119.

54 Ibid., p. 121.

55 Schmid, W., En busca de un nuevo arte de vivir. La pregunta por el fundamento y la nueva fundamentación de la ética en Foucault (G. Cano, trad.), Pre-textos, Valencia 2002, p. 77. 
La construcción de la historia de estos enfrentamientos, desplazamientos y reconquistas recíprocas, que Foucault ha descubierto con el estudio de la Grecia antigua, suscita su anunciado interés en la figura de Montaigne. En el fragmento citado anteriormente del debate en el Departamento de Francés de la Universidad de California en Berkeley, el autor señalaba algo de especial relevancia: la noción de ensayo en Montaigne, de prueba de sí, está más próxima del examen de conciencia estoico, donde la prueba tiene como fin saber de lo que uno es capaz, conocer la libertad de la que se dispone y la dependencia a la que se está sometido, que de la búsqueda de una verdad oculta en el fondo de uno mismo ${ }^{56}$. El desciframiento de sí, que no está presente en Montaigne, es precisamente lo que define la hermenéutica de sí que comienza con el cristianismo. Son dos las principales obligaciones que aparecen con él: la obligación de creer y la obligación de descubrir y decir la verdad sobre uno mismo; hermenéutica del texto y hermenéutica de si $^{57}$. Esta última surge gracias a una mutación en las técnicas de sí griegas y romanas que no tenían dicho objetivo. Ambas obligaciones forman parte del régimen obediencia propio del poder pastoral y el movimiento que inicia Lutero y que define a la Reforma tiene como objetivo establecer una nueva relación entre ambas y liberarlas del dominio de la Iglesia ${ }^{58}$. Sin embargo, dado que no modifica la estructura que dibujan estas dos obligaciones, la Reforma protestante no deja de ser una revolución pastoral $^{59}$. Ahora bien, en el marco de la reactivación de temas y prácticas antiguas propia del Renacimiento, de la Reforma y la Contrarreforma ${ }^{60}$, Montaigne marca una cesura en la medida en que la hermenéutica de sí no está presente en su obra, lo que abre la posibilidad de una nueva estética de la existencia con características propias. Siguiendo de nuevo a Deleuze, podríamos decir que como consecuencia de un conflicto autodestructor de distintos paradigmas "coactivos» se abre un espacio en el que activar unas «reglas facultativas ${ }^{61}$. En el esquema foucaultiano, Montaigne representa un momento efímero en el que aparece una nueva práctica de libertad que desborda las técnicas pastorales de poder, de ahí su interpretación positiva del autor bordelés. Sin embargo, Descartes marca la clausura de este breve episodio: tras algunas oscilaciones, la hermenéutica de sí volvió a triunfar ${ }^{62}$. El «coup cartésien» transforma la prácti-

56 Foucault, M., Qu'est-ce que la critique? Suivi de La culture de soi, p. 169.

57 Foucault, M., Mal faire, dire vrai. Fonction de l'aveu en justice. Cours de Louvain, 1981, p. 90 .

58 Ibid., pp. 90, 165-166. Esta temática también es abordada en Foucault, M., L'origine de l'herméneutique de soi. Conférences prononcées à Dartmouth College, 1980, pp. 109-110.

59 Foucault, M., Sécurité, territoire, population. Cours au Collège de France (1977-1978), Gallimard, París 2004, p. 153.

${ }^{60}$ Foucault, M., «À propos de la généalogie de l'éthique: un aperçu du travail en cours», en: Dits et écrits vol. IV (1980-1988), Gallimard, París 1994, p. 628.

${ }^{61}$ Deleuze, G., o.c., p. 119.

62 Foucault, M., L'origine de l'herméneutique de soi. Conférences prononcées à Dartmouth College, 1980, p. 89. Diversas investigaciones han discutido la interpretación foucaultiana del significado de Descartes en la historia de la filosofía, para lo que han intentado actualizar 
ca de sí montaigniana en una hermenéutica de sí en la medida en que el sujeto encuentra el fundamento del conocimiento científico, la evidencia, al escrutar su interior ${ }^{63}$. A partir de entonces, el fundamento de la hermenéutica de sí no podía ser el sacrificio de sí cristiano, sino que pasó a ser el sí mismo teórico y práctico. Se abre la puerta así al desarrollo y surgimiento de las ciencias humanas en los siglos posteriores. Y este intento de construir un sí mismo positivo como fundamento de la hermenéutica de sí es lo que Foucault denomina el «antropologismo» propio de nuestra cultura, que no es más que el correlato de unas tecnologías de poder ${ }^{64}$.

En el estudio de la aparición de la «gubernamentalidad», ligada especialmente a la razón de Estado como nuevo arte de gobierno, y de las tecnologías que le van aparejadas, Foucault señala que esta transformación con respecto al poder pastoral se produce precisamente junto con el surgimiento de la episteme clásica ${ }^{65}$. Tras la expulsión de la locura del ámbito de lo razonable y la transformación de la ascesis de sí en práctica fundadora de conocimiento, la evidencia constituye el punto de unión entre sujeto y verdad. El mundo de semejanzas propio del Renacimiento da paso a un cosmos cognoscible y ordenable de forma exhaustiva mediante el análisis de identidades y diferencias, la actividad propia del espíritu ${ }^{66}$. Es en el marco de la episteme clásica y sus transformaciones posteriores ${ }^{67}$ donde van a comenzar a gestarse tanto las

dicha lectura desde un punto de vista montaigniano, es decir, subrayando la importancia de la incertidumbre y de la "autoexposición» en el texto cartesiano. C fr. LlinÀs, J., «La philosophie comme forme de vie : un Descartes montaignien ?» en: Montaigne Studies, 25, 2017, pp. 169-176; Meleny, H., Writing cogito: Montaigne, Descartes, and the institution of the modern subject, State University of New York Press, Albany 1997. Por otro lado, no se ha dejado de incidir en el carácter «violento» de la operación cartesiana. Cfr. FuJita, K., «La naissance du cogito», en: Lorenzini, D., y Sforzini, A., (eds.), Un demi-siècle d'Histoire de la Folie, Éditions Kimé, París 2013, pp. 103-116.

${ }^{63}$ Ibid., pp. 119-121. En esta línea, para un estudio de la concepción de la subjetividad y de la función de la evidencia en Montaigne y Descartes, así como de las consecuencias que de ellas se derivan para el pensamiento de ambos autores, especialmente críticas en el caso del primero, $c f r$. De Souza, T., «Regard sur soi, l'esprit qui connaît : figures de la subjectivité chez Montaigne et Descartes» en: Montaigne Studies, 25, 2013, pp. 31-38; Paganini, G., "Les transformations du doute de Montaigne à Descartes», Montaigne Studies, 25, 2013, pp. 39-48; Brahami, F., «Conscience et raison: l'humanité chez Montaigne et Descartes», en: Panichi, N., Ragghianti, R., y Savorelli, A., (eds.), Montaigne contemporaneo, Edizioni Della Normale, Pisa 2011, pp. 159-174.

64 Ibíd. p. 90.

65 Foucault, M., Sécurité, territoire, population. Cours au Collège de France (1977-1978), p. 242.

66 Foucault, M., Les mots et les choses, p. 69.

67 Hay que tener en cuenta la importancia que en este proceso tiene la episteme moderna, en la que el hombre se constituye como doblete-empírico trascendental y surge un discurso virtual de lo no-conocido que incita sin cesar al hombre al conocimiento de sí. $C f r$. Foucault, M., Les mots et les choses, pp. 333-339. Recientes investigaciones han reactivado la discusión sobre las bases documentales y la construcción teórica de las epistemes en Foucault: $c f r$. Villacañas, J., «Foucault 1964» en: Res Publica, 24, 2010, pp. 11-38. 
tecnologías disciplinarias, que encierran, reglamentan todo detalle, establecen una distribución entre lo permitido y lo prohibido para finalmente vencer a la realidad, como los dispositivos de seguridad, que promocionan el movimiento en lugar de valorar el detalle, dejan hacer como un hecho natural, sustituyendo la división binaria entre lo permitido y lo prohibido por una media óptima y unos límites de lo aceptable, accesibles estadísticamente ${ }^{68}$. En conclusión, una profunda transformación de la cultura occidental que tiene como uno de sus movimientos otro «recurso» de las técnicas de dominación a técnicas usadas en las relaciones de sí consigo. También Deleuze ha señalado esta confrontación en la que el Estado disciplinario y las nuevas formas de saber se fundamentan en el conocimiento y el control de la subjetividad, de un sí mismo cuya interioridad puede y debe ser conocida ${ }^{69}$.

Por último, es necesario señalar que, además de los intercambios y transformaciones entre los ejes del poder, el saber y la subjetivación que se acreditan en estos periodos, el interés de Foucault por la Grecia antigua o por algunos desarrollos del Renacimiento, no se debe a que encuentre en ellos una forma de vida cuya restauración desee, o una verdad intemporal que se abra paso en distintos momentos de la historia, algo que sería ajeno a los principios elementales de su filosofía ${ }^{70}$. La razón es que estima que la moral entendida como obediencia a un sistema de reglas es ya impracticable ${ }^{71}$ y ante esta situación solo cabe la vía de una estética de la existencia para cuyo desarrollo el estudio de la moral griega o de las prácticas de sí renacentistas pueden iluminarnos de forma oblicua.

\section{Montaigne, el ensayo y la ontología crítica}

En la conferencia de 1983 La cultura de sí, Foucault establece explícitamente el nexo entre la ontología de la actualidad y su estudio de la cultura de sí antigua. El autor sitúa su propia tarea filosófica en una de las dos tradiciones que, a su juicio, Kant inaugura y que se imponen a todo quehacer filosófico. Por un lado se encuentra la analítica de la verdad u ontología formal de la verdad, es decir, la investigación de las condiciones del conocimiento verdadero. Por otro lado, la pregunta por la Aufklärung abre por primera vez la pregunta por nuestra propia actualidad, por el campo de experiencias que nos son posibles. Esta última es definida por Foucault como una ontología de la actualidad o una

68 Foucault, M., Sécurité, territoire, population. Cours au Collège de France (1977-1978), pp. 58-59.

${ }^{69}$ Deleuze, G., o.c., p. 120.

70 En otro hilo argumental, Foucault llega a considerar la Antigüedad como un «profundo error» por haber buscado obstinadamente un estilo de existencia y haberse esforzado a la vez por hacerlo común a todos. Cfr. Foucault, M., «Le retour de la moral», en: Dits et écrits (vol. $I V)$, Gallimard, París 1994, p. 698. La traducción es nuestra.

71 Foucault, M., "Une esthétique de l'existence», en: Dits et écrits (vol. IV), Gallimard, París 1994, p. 732. 
ontología crítica de nosotros mismos ${ }^{72}$. Y para el estudio de nuestra relación con la actualidad, Foucault declara haber considerado necesario el examen de tres conjuntos de relaciones: con la verdad, con la obligación y con nosotros mismos y con los otros ${ }^{73}$. En la investigación de estas últimas, como ya se ha indicado, arribó a la antigüedad clásica en la que identifica el desarrollo de una "cultura de sí». En el texto proporciona cuatro características que permiten distinguir el esplendor de esta cultura en los dos primeros siglos de nuestra era de la interpretación del precepto epimeleia heautou en el Alcibíades de Platón: la inquietud de sí define un tipo de relación permanente consigo mismo y no un momento puntual ligado a una preparación pedagógica, es una relación crítica consigo mismo, implica la aceptación de la autoridad del maestro y las prácticas que conlleva van más allá de la pura contemplación del alma. La función crítica implica que la cultura de sí no solo debe permitir la adquisición de conocimientos, sino desaprender las opiniones falsas y los malos hábitos ${ }^{74}$. Por tanto le es indisociable un aspecto corrector, especialmente presente en los cínicos y estoicos, que permita al sujeto dejar de ser lo que le impide alcanzar la autonomía frente a los acontecimientos del mundo ${ }^{75}$. Ahora bien, el sujeto se enjuicia con la intención de calibrar el grado de cumplimiento de los principios buscados que su conducta muestra. No se trata de reprocharse ni de castigarse, sino de solucionar problemas técnicos ${ }^{76}$.

La crítica es imperativa para cumplir con la ya mencionada función de la ascesis filosófica: la transformación del logos en ethos. Si el ascetismo cristiano, al convertir el conocimiento y la comunicación de sí en la piedra angular de la hermenéutica de sí, se busca la «objetivación de sí en un discurso de verdad», en la cultura de sí la «subjetivación del discurso de verdad» es el fin ${ }^{77}$. Es necesario apropiarse de la verdad, convertirla en el propio modo de ser, encarnarla. Y como si se tratara de un aleta, el sujeto ha de ejercitarse en los preceptos que le permitirán conseguir una vida a salvo de los envites de la realidad, en la verdad que quiere expresar en su estilo de vida ${ }^{78}$.

La cultura de sí, además de representar un periodo de esplendor donde la relación del sujeto consigo mismo es ejercida como práctica de libertad, adquiere una importancia fundamental en la obra de Foucault gracias a su componente crítico. Este la convierte en un momento cardinal en la genealogía de lo que el autor llama el «ethos filosófico», entendido como "crítica permanente de

72 Foucault, M., Qu'est-ce que la critique? Suivi de La culture de soi, p. 84.

73 Para un estudio de la noción de experiencia en Foucault y de su construcción a través de estos tres ejes, $c f r$. Morey, M., "Michel Foucault: una política de la experiencia», en: Escritos sobre Foucault, Sexto Piso, Madrid 2014, pp. 145-162.

74 Foucault, M., Qu'est-ce que la critique? Suivi de La culture de soi, p. 91-93.

75 Foucault, M., L'herméneutique du sujet. Cours au Collège de France (1981-1982), p. 90.

76 Ibid., p. 463.

77 Ibid., p. 316.

78 Ibid., p. 213. 
nuestro ser histórico» ${ }^{79}$. En la reflexión sobre su propio trabajo, el autor define su tarea como determinada históricamente por la Ilustración y la pregunta por la actualidad que con ella se abre. Define su tarea como la de una ontología crítica en la que tiene lugar la crítica de lo que somos, en tanto que investigación sobre los límites propios, y la prueba de su posible transgresión ${ }^{80}$. A diferencia de la kantiana, esta no se ocupa de los límites necesarios del conocimiento ni de las estructuras trascendentales, sino de los acontecimientos contingentes que nos han llevado a constituirnos, para así entrever la posibilidad de dejar de ser lo que somos ${ }^{81}$. En consecuencia, como ha visto De la Higuera, la «prueba de eventualización» que impone la filosofía foucaultiana a las prácticas o a las formas de pensar, para descubrir los nexos de poder y saber que permiten su aceptabilidad, supone también una eventualización del discurso filosófico. Así, el autor acepta la disolución de su propia palabra porque «[n]o se trata de decir lo que ha pasado, sino de liberarnos de ello. La ficción histórica tiene la finalidad de producir efectos reales sobre nuestro presente y, en esa medida, adquiere una verdad que no poseía con anterioridad ${ }^{82}$. La empresa cognoscitiva se subordina a una tarea mayor destinada a la transformación del propio ethos. Una transformación ya no guiada por preceptos morales que nos equipen para la consecución de la autonomía, sino por el mismo movimiento que relata la constitución genealógica de la realidad.

Pero, además de la cultura de sí antigua, la genealogía del ethos filosófico también tiene como uno de sus episodios clave la aparición de la "actitud crítica» en los siglos XV y XVI. Esta coincide con la transformación que supone la aparición de la «gubernamentalidad», ya mencionada. Foucault documenta la expansión de la preocupación por el gobierno, que desborda el foco religioso y atraviesa las inquietudes civiles, y su especificación en dominios concretos: cómo gobernar a los niños, cómo gobernar al cuerpo o cómo gobernar al Estado. De forma simultánea surge también la pregunta sobre cómo no ser gobernado, no en absoluto, sino de tal forma. Como rival de estas artes de gobierno nace la necesidad de enfrentarse constantemente a ellas para explotar sus límites precisos en oposiciones concretas. De esta forma, la crítica opera en la relación del sujeto con la verdad para llevar a cabo una función de «desujeción»" ${ }^{83}$. Y teniendo en cuenta que la publicación de los Ensayos se extendió desde 1580 hasta la edición póstuma de 1595, es evidente que contribuye y forma parte de la activación de esta actitud crítica que posteriormente sufrirá nuevas rupturas y torsiones, como es el caso de la kantiana, y que por tanto también representa una instancia ineludible para la genealogía de la tradición de pensamiento en

79 Foucault, M., «Qu'est-ce que les Lumières?», en: Dits et écrits (vol. IV), Gallimard, París 1994, p. 571.

$80 \quad$ Ibíd., p. 577.

81 Ibíd., p. 572.

82 De la Higuera, J., «Estudio preliminar», en: Foucault, M., Sobre la Ilustración, Tecnos, Madrid 2007, pp. 42-44.

${ }^{83}$ Foucault, M., Qu'est-ce que la critique? Suivi de La culture de soi, pp. 36-39. 
la que el propio Foucault se sitúa ${ }^{84}$. No es casual que el autor llame a la actitud crítica el «arte de la inservidumbre voluntaria» ${ }^{85}$, una referencia velada al Discurso de la servidumbre voluntaria de Étienne de la Boétie, unido a Montaigne por una amistad ya legendaria.

Tras siglos de una filosofía que pretendía describir el orden metafísico que regía el mundo, aparece en escena lo que Montaigne define como «un filósofo impremeditado y fortuito ${ }^{86}$, cuya reflexión está presidida por la constatación de que no hay comunicación con el ser. Pero ¿qué quiere decir esto? En un primer momento, parecería que nos encontramos ante una mirada impotente, ante un epílogo del pirronismo antiguo, incapaz de justificar su propia postura desde el momento en el que afirma, incurriendo en autocontradicción performativa, que no existe la verdad. ¿Cómo es posible sostener una proposición, susceptible de ser verdadera o falsa, si no podemos conocer la verdad o directamente no existe? Sin embargo, el escepticismo del ensayo supera este problema a partir de una nueva concepción de la verdad y el emblema de Montaigne nos da la pista fundamental de la revolución filosófica que opera en esta nueva forma filosófica. La máxima «Que sais-je?» es una pregunta y, en consecuencia, no puede ser ni verdadera ni falsa. Por esta razón Merleau-Ponty sostiene que «Montaigne empieza por enseñar que toda la verdad se contradice y quizás acaba por reconocer que la contradicción es verdad $»^{87}$. Así, lo que encontramos en los Ensayos, antes que un reflejo de la incapacidad epistemológica del ser humano, es el rechazo de una concepción unitaria del ser, de una visión parmenídea del mundo, y la apertura a una ontología del devenir. A causa de la inestabilidad de lo real, y en consecuencia de sí mismo, Montaigne se pone a prueba, se ensaya: «si mi alma pudiera asentarse, no haría ensayos» ${ }^{88}$. La consecuencia del socavamiento de la metafísica escolástica de raigambre aristotélica será la

84 Para un estudio de las diferencias y similitudes entre el ensayo moderno y el contemporáneo, $c f r$. DE LA Higuera, J., «El lugar del ensayo», en: García Casanova, J., (coord.), El ensayo, entre la filosofía y la literatura, Comares, Granada 2000, pp. 33-66. La influencia de los Ensayos en la gestación de los discursos críticos ha sido analizada en Guerrier, O., «De l'œuf à la poule. L'essai montaignien : la vie, la pensée, l'écriture», en: Née, P. (coord.), Naissance de la critique littéraire et de la critique d'art dans l'essai, Classiques Garnier, París 2019, pp. 35-50.

${ }_{85}$ Foucault, M., Qu'est-ce que la critique? Suivi de La culture de soi, p. 39. Para una investigación del nexo entre los tres autores, $c f r$. Schachter, M., «'Qu'est-ce que la critique?' La Boétie, Montaigne, Foucault», en Zalloua, Z., (ed.), Montaigne After Theory, Theory After Montaigne, University of Washington, Press, Seattle 2009, pp. 122-141.

86 II, 12, 578. Las citas de los Ensayos se harán siguiendo la edición de Balsamo, J., Magnien-Simonin, C., y Magnien, M., Les Essais, Gallimard, París 2007. El número romano hará referencia al libro, el primer arábigo al capítulo y el segundo arábigo a la página. La traducción es nuestra.

87 Merleau-Ponty, M., «Lectura de Montaigne», en: Signos (C. Martínez y G. Oliver trad.), Seix Barral, Barcelona 1960, p. 247.

88 III, 3, 845 . 
crítica del dogmatismo de las morales rigoristas que conllevan una mortificación de sí y posiciones políticas extremas ${ }^{89}$.

Es imprescindible volver a señalar que si los Ensayos de Montaigne forman parte de la genealogía de la actitud crítica ello implica un desplazamiento en su lectura. No hay que ver en ellos una operación de autoconocimiento, el intento del autor de objetivarse en un discurso que le permita conocerse, lo que Foucault denomina una «hermenéutica de sí». Antes bien, en lugar de una operación de objetivación de sí, en la obra hay un trabajo de subjetivación de sí, la tentativa de darse a sí mismo una forma, imprimir sobre sí un estilo. A este respecto es reveladora la descripción que ofrece Foucault del ejercicio que supone la meditación: no se trata de un juego del sujeto sobre su pensamiento, sino del pensamiento sobre el propio sujeto ${ }^{90}$. Por tanto, la pregunta que la genealogía lanza sobre la filosofía de Montaigne no se refiere a los contenidos específicos de su obra, como la educación, la amistad o los caníbales; sino sobre la operación mediante la cual transforma el logos en ethos: ¿qué modo de ser produce? Si Montaigne aconseja que el lector no fije su atención en la materia que aborda, sino en la manera en la que las trata ${ }^{91}$, ¿cuál es dicha manera? ¿Qué criterios utiliza para dicha transformación? ¿Cómo se convierte, a través de la escritura, su interpretación de la realidad en un estilo de existencia? ${ }^{92}$. Es el acto de subjetivación el que ha de guiar la comprensión de los Ensayos, y en este marco han de ser desarrollados los estudios sobre el gobierno de sí o la parresía ${ }^{93}$. En esta obra encontramos una práctica de constitución de sí que implicó su escritura y al que invita su lectura.

$89 C f r$. ScoralicK, A., "Vivre comme Socrate. La notion d'ordre et la morale de l'homme médiocre dans les derniers Essais de Montaigne» en: Bulletin de La Société Internationale Des Amis de Montaigne, 64(2), 2016, pp. 71-85; Calnoun, A., Montaigne and the Lives of the Philosophers. Life Writing and Transversality in the Essais, University of Delaware Press, Newark 2015; Nadeau, C., "Montaigne contre l'éthique de la vertu», en: Gontier, T., y Mayer, S., (eds.), Le Socratisme de Montaigne, Classiques Garnier, París 2010, pp. 173-187.

90 Foucault, M., L'herméneutique du sujet. Cours au Collège de France (1981-1982), p. 340.

91 II, 10, 428.

92 A este respecto, la lectura foucaultiana que aquí se intenta reconstruir entronca con investigaciones recientes sobre Montaigne o sobre el ensayo centradas en la operación sobre sí. Cfr. Force, P., "Montaigne and the Coherence of Eclecticism» en: Journal of the History of Ideas, 70(4), 2009, pp. 523-544; SÈve, B., Montaigne. Des règles pour l'esprit, Presses Universitaires de France, París 2007; Cerezo, P., «El espíritu del ensayo», en: García Casanova, J., (coord.), El ensayo, entre la filosofía y la literatura, Comares, Granada 2000, pp. 1-32.

93 A raíz de la aparición de los textos del último Foucault, se ha rastreado la presencia de las nociones antiguas relacionadas con el cuidado de sí en la obra de Montaigne. Cfr. LLINÀs, J., «La cuestión animal y el gobierno de sí. Montaigne, Descartes y Derrida» en: Ingenium, Revista Electrónica de Pensamiento Moderno y Metodología en Historia de la Ideas, 11, 2017, pp. 87-102; Guerrier, O., "Le Socrate de Foucault et le "socratisme" de Montaigne. Autour de la Parrhêsia», en: Gontier, T., y Mayer, S., (eds.), Le Socratisme de Montaigne, Classiques Garnier, París 2010, pp. 57-69; Krause, V., "Confession or Parrhesía? Foucault after Montaigne», en: Zalloua, Z., (ed.), Montaigne After Theory, Theory After Montaigne, University of Washington Press, Seattle 2009, pp. 142-160; Luque, Á. «Literature of the Self in Foucault: Parrhesia and Autobiographical Discourse» en: CLCWeb: Comparative Literature and Culture, 20.4, 7, 2018; 
Las escasas ocasiones en las que Foucault menciona a Montaigne permiten reconstruir una lectura que, en relación con contenidos especialmente relevantes de su pensamiento, sitúan al autor de los Ensayos en una posición privilegiada. En un momento en el que la razón todavía no se ha pertrechado frente a su enemigo irrazonable y el espíritu no se ha convertido en una instancia analizadora de identidades y diferencias, se reactiva bajo nuevas coordenadas la necesidad de efectuar sobre sí mismo una transformación mediante el pensamiento. Aun siendo imposible saber si Foucault hubiera entrado a fondo en el estudio de la obra de Montaigne, de la lectura aquí ensayada es posible concluir que la importancia del pensamiento del autor de los Ensayos es profundamente subrayada. En él se halla uno de los hitos fundamentales de nuestra comprensión de la filosofía en tanto que actividad dedicada a la transformación de nuestro ser histórico. El estudio de la operación que en ellos tiene lugar no solo es imprescindible para una mejor comprensión de la génesis de la modernidad, sino que se revela fundamental para una iluminación oblicua de la ontología del presente.

Universidad de Granada

BELTRÁN JimÉnEZ Villar

beltranjimenez@ugr.es

[Artículo aprobado para publicación en febrero de 2020]

CARneIro, A., «Spiritual exercises and parrhesia in Montaigne's Essays» en: Revista de filosofía Aurora, 23(32), 2011, pp. 113-129; Allen, S., «The Cultivated Self: Self Writing, Subjectivity, and Debate» en: Rhetoric Review, 29(4), 2010, pp. 364-378. 